\title{
LETTER
}

\section{Pathological changes in a patient with acute respiratory distress syndrome and H7N9 influenza virus infection}

\author{
Qiang Guo ${ }^{1 *+}$, Jian-an Huang ${ }^{1 \dagger}$, Daguo Zhao ${ }^{1+}$, Jun Jin ${ }^{1}$, Shenlang Liu ${ }^{1}$ and Dustin R Fraidenburg ${ }^{2}$
}

There are very few data regarding pathological changes in patients with severe pneumonia and acute respiratory distress syndrome (ARDS) from avian influenza A (H7N9) virus. We present the case of a 73-year-old woman with a history of hypertension and prior cerebrovascular accident who was admitted to the hospital complaining of dyspnea, cough with scant hemoptysis, and fever. A history of live poultry exposure was elicited during an initial interview.

Hypoxic respiratory failure led to endotracheal intubation and mechanical ventilation on the first day of hospitalization. Significant lymphopenia was also noted upon admission. Infection with influenza A (H7N9) virus was confirmed from a tracheal aspirate sample using polymerase chain reaction assays. Thoracic imaging revealed diffuse bilateral infiltrates that were associated with impaired gas exchange and a diagnosis of ARDS. Despite the administration of oseltamivir and peramivir, the patient continued to deteriorate. Bronchoscopy with bronchoalveolar lavage specimens suggested possible co-infection with four potential pathogens (Pseudomonas aeruginosa, Flavobacterium meringosepticum, Staphylococcus haemolyticus, and Candida albicans) and empiric antimicrobial therapy was begun.

Computed tomography revealed ground glass opacities and consolidation in both lungs. High positive endexpiratory pressure $\left(>7 \mathrm{cmH}_{2} \mathrm{O}, 27\right.$ days total) and high plateau pressures $\left(>30 \mathrm{cmH}_{2} \mathrm{O}, 27\right.$ days total) were necessary as rescue therapies in order to maintain the goal of

\footnotetext{
* Correspondence: ggqq1018@hotmail.com

${ }^{\dagger}$ Equal contributors

${ }^{1}$ Department of Medicine, Respiratory, Emergency and Intensive Care Medicine, The First Affiliated Hospital of Soochow University, 188 Shizhi Street, Suzhou 215006, China

Full list of author information is available at the end of the article
}

partial pressure of arterial oxygen $>55 \mathrm{mmHg}$. Mechanical ventilation was complicated by right pneumothorax on day 9 , and chest tube drainage was performed that resulted in a persistent air leak. Severe sepsis and ARDS was complicated by stress cardiomyopathy and the patient expired on hospital day 27.

A limited autopsy was performed and small samples of right upper lung tissue were obtained, fixed, and embedded. The samples were used for hematoxylin and eosin staining, Masson staining, and smooth muscle actin immunohistochemistry staining. Histopathological evaluations revealed diffuse alveolar damage characterized by edema, hyaline membranes, inflammation, and fibrosis. Fibrin thrombus within the vascular lumen, inflammatory infiltrate below the endothelium, and necrosis of bronchiolar walls were also present (Figure 1). It is notable that these findings show similarity to the pattern observed in patients of avian influenza (H5N1) [1,2] and 2009 influenza A (H1N1) [3,4]. Immunohistochemistry showed severe fibrosis and thickened distal pulmonary arteries in the samples obtained (Figure 1).

This case illustrates that, in addition to the primary viral pneumonia, complications including ARDS and bacterial co-infections may contribute to diffuse alveolar damage, severe fibrosis, and thickened distal pulmonary arteries as the most significant and consistent finding observed with severe H7N9 virus infection. More studies are needed to further characterize the pathogenesis and to determine effective treatment strategies for severe respiratory illness caused by H7N9 virus infection. 


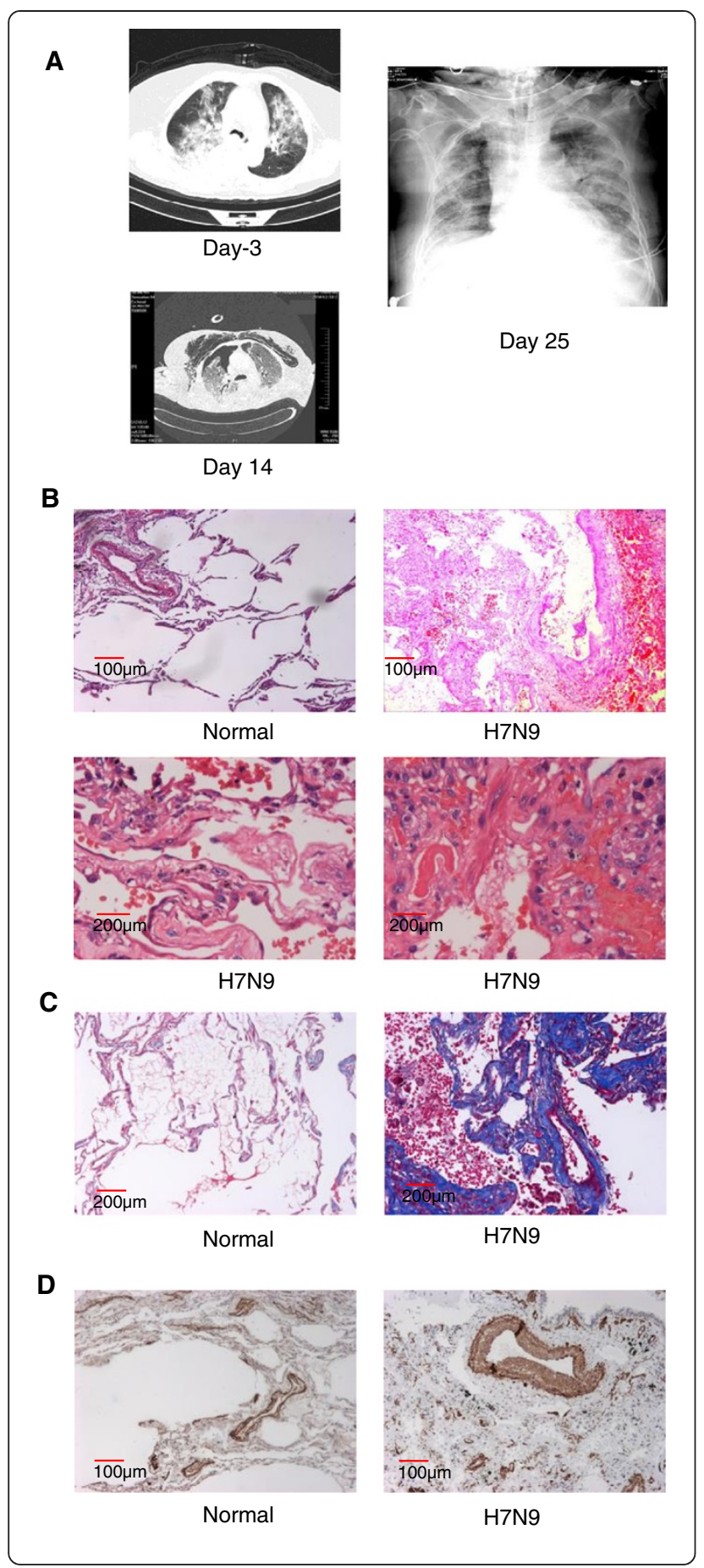

Figure 1 Micrographs of lung tissue, pathophysiology, and treatment from a patient with acute respiratory distress syndrome and H7N9 influenza. (A) Two computed tomography images ( 3 days before admission (day -3 ) and 14 days after admission (day 14)) and the patient's last X-ray image (2 days before death (day 25)). (B) Hematoxylin and eosin staining from a normal sample and the H7N9 patient's lungs. Diffuse alveolar damage, including edema, hyaline membranes, and inflammation, were seen in these images. (C) Masson staining in normal lungs and the H7N9 patient's lungs. Severe fibrosis (blue) was seen in the H7N9 patient. (D) Immunohistochemical staining with smooth muscle actin in normal lungs and the H7N9 patient's lungs. Thickened distal pulmonary arteries were seen in the H7N9 patient.

\section{Abbreviation}

ARDS: Acute respiratory distress syndrome.

\section{Competing interests}

The authors declare that they have no competing interests.

\section{Acknowledgements}

The patient's son provided written informed consent for publication. This work was supported by grants from the National Natural Science Foundation of China (NSFC81300040), The Natural Science Foundation of Jiangsu Province (BK20141184), and the China National Clinical Key Subject and Application Foundation of Suzhou China (SYS201336).

\section{Author details}

'Department of Medicine, Respiratory, Emergency and Intensive Care Medicine, The First Affiliated Hospital of Soochow University, 188 Shizhi Street, Suzhou 215006, China. ${ }^{2}$ Department of Medicine, Division of Pulmonary, Critical Care, Sleep and Allergy Medicine, University of Illinois at Chicago, Chicago, IL 60612, USA.

Published online: 05 December 2014

\section{References}

1. Gu J, Xie Z, Gao Z, Liu J, Korteweg C, Ye J, Lau LT, Lu J, Zhang B, McNutt MA, Lu M, Anderson VM, Gong E, Yu AC, Lipkin WI: H5N1 infection of the respiratory tract and beyond: a molecular pathology study. Lancet 2007, 370:1137-1145.

2. Korteweg C, Gu J: Pathology, molecular biology, and pathogenesis of avian influenza A (H5N1) infection in humans. Am J Pathol 2008, 172:1155-1170.

3. Wun JS, Dianna M, Amy M, Marlene D, Patricia A, Julu B, John S, Lindy L, Mitesh P, Brigid B, Patricia G, Tara J, Chalanda S, Jeanine B, Jeltley M, Elizabeth W, Dominique R, Rongbao G, Cynthia S, Heather J, Maureen M, Cynthia S, Charles H, Ann S, Clifton D, Christopher P, Timothy M, Sherif R: 2009 pandemic influenza $A$ (H1N1) pathology and pathogenesis of 100 fatal cases in the United States. Am J Pathol 2010, 177:166-175.

4. Virgilia S, Juan S, Alberto D, Patricia V, Oralia T, Alfonso R, Ana C, Patricia A, Francisco N: Pathological changes associated with the 2009 H1N1 virus. N Engl J Med 2009, 2009:2001-2003.

doi:10.1186/s13054-014-0666-y

Cite this article as: Guo et al:: Pathological changes in a patient with acute respiratory distress syndrome and H7N9 influenza virus infection. Critical Care 2014 18:666. 